\title{
INDICADORES DE SUSTENTABILIDADE EMPRESARIAL E GESTÃO ESTRATÉGICA
}

\section{Anderson Américo Alves Cantarino}

\section{Martha Macedo de Lima Barata}

Emilio Lèbre La Rovere

\section{RESUMO}

A partir da constatação de que as empresas, para serem sustentáveis, necessitam gerenciar também as variáveis ambiental, social, de saúde e de segurança do trabalhador, os autores propõem critérios para definir, implementar e relatar indicadores que possibilitem, aos gestores de empresa, avaliarem seu desempenho nas mencionadas variáveis, ao longo do tempo e em comparação com outras empresas. A metodologia utilizada baseou-se em levantamento de informações, avaliação bibliográfica e consolidação da experiência acumulada em estudo de caso de empresa do setor de petróleo. Em análise dos relatórios de sustentabilidade atualmente publicados por empresas do setor de petróleo os autores detectaram que estas usam indicadores que denotam uma atitude reativa das mesmas, o que dificulta sua mudança de postura e, portanto a sua efetiva sustentabilidade.

Palavras-chave: Sustentabilidade empresarial. Indicador de desempenho. Gestão ambiental empresarial

\begin{abstract}
The business sector observed that in order to be sustainable it is necessary to manage also their social, environmental, health and security aspects, that is why the authors propose a technique based on setting indicators for helping business sector in achieving a good performance on those aspects. Based on bibliographic research and experience in working with petroleum management, they present how business sector should identify, implement and report the sustainable indicators. Analyzing sustainable reports published by oil companies the authors identified that they were using indicators showing a reactive attitude which brings obstacles to their sustainability.
\end{abstract}

Keywords: Business sustainability. Performance indicator. Business environmental management. 


\section{INTRODUÇÃO}

0 gerenciamento da reputação da empresa é elemento central da gestão corporativa. Até meados da década de 1990 o desenvolvimento sustentável era abordado no âmbito empresarial através de uma agenda verde. Durante a década de 1990 tornaram-se comuns acusações de exploração, pelo uso da mão de obra infantil, pelo massacre dos sindicatos, dentre outros, que induziram empresas a incorporarem também a dimensão social em sua gestão. Observa-se que esta é mais relevante para empresas situadas em países que ainda enfrentam grandes desafios sociais (Hollyday, 2003; La Rovere; Barata, 2003).

Observa-se que no cenário atual, as partes interessadas ${ }^{i}$ têm requerido que a gestão das empresas considere as questões éticas e sociais com o mesmo rigor aplicado às questões econômicas e ambientais. Assim, em conjunto com os requisitos de desempenho ambiental e econômico, a responsabilidade social forma o tripple bottom line ii $^{\mathrm{i}}$ Neste cenário, a Sustentabilidade Empresarial, isto é, a gestão lucrativa da empresa ao longo do tempo pressupõe, dentre outras, a habilidade de simultaneamente ampliar a atividade econômica, reduzir os impactos ambientais e contribuir para a melhoria da qualidade de vida humana (Barata, 2003). A crescente importância destas outras variáveis na determinação do valor da empresa pode ser percebida pela criação em 1999 do Índice Dow Jones de Sustentabilidade (DJSGI), que define a sustentabilidade de acordo com os critérios econômicos, sociais e ambientais, incorporando aos tradicionais valores empresariais os princípios da tecnologia inovativa, do bem estar social e da responsabilidade ambiental, dentre outros. Em 2000 a capitalização total do mercado do DJSGI foi de 4,3 trilhões de dólares. Destaca-se também estudo realizado por Stanley J. Feldman, Peter Soyka e Paul Ameer da ICF Kaiser International, Inc (1997) que desenvolveram modelo para entender como as atividades ambientais corporativas são traduzidas em mudanças no valor de mercado da empresa. 0 modelo foi aplicado em mais de 300 empresas nos EUA, e os resultados indicaram que as melhorias ambientais reduziam o risco da empresa e possibilitavam um crescimento do preço da ação da empresa em cerca de 5\%. Estudo realizado por Barata (2001) apresentou que empresas que pretendam ter suas ações comercializadas através das regras do Securities and Exchange Commission (SEC) necessitavam demonstrar a saúde ambiental de sua empresa, geralmente através de notas explicativas na sua demonstração financeira. Segundo pesquisa realizada pela UNCTAD (1998), o mesmo ocorria durante operações comerciais na Dinamarca, na Holanda, na Noruega e na Tailândia. Na Inglaterra, apesar de não ser exigido, havia incentivo para que empresas com mais de 250 empregados o fizessem. Na ocasião a variável ambiental ainda não era objeto de avaliação para o mercado acionário brasileiro, havia apenas estudos acadêmicos sendo feitos para o subsidiar a Comissão de Valores Mobiliários (CVM) no requerimento de Balanços Ambientais (Grillo Ramos, 1999); (Ferreira,1998); (Ferreira, 1999); (Guarnieri,2001);(Ribeiro,1992), (Ribeiro, 1998) e (Barata,2002). A forma mais adequada para a demonstração desta responsabilidade nas empresas também tem sido objeto de estudo na Europa, EUA , América Latina. Na segunda metade da década de 90 diversas leis estaduais e municipais incentivaram a publicação do Balanço Social das empresas no Brasiliii.

\footnotetext{
i Empregados, acionistas, competidores, investidores, clientes, etc.

ii Representa a avaliação do desempenho de uma determinada empresa através do julgamento dos critérios de eficiência econômica, proteção ambiental e responsabilidade social.

iii Elas deram prosseguimento ao projeto de Lei no. 3116/97, que pretende estabelecer a obrigatoriedade de apresentação do Balanço Social para entidades públicas e privadas. Ocasião em que o IBASE lançou o primeiro modelo de Balanço Social para o Brasil.
} 
Práticas já estabelecidas de sustentabilidade corporativa permitem às empresas reduzir custos, aumentar a fidelidade dos funcionários e clientes, criar novos mercados e reduzir as chances de serem surpreendidos por algum tipo de ônus indesejável e inoportuno. Entretanto, apesar da motivação pela Sustentabilidade Empresarial, o gestor da empresa nem sempre dispõe de instrumento que lhe possibilite visualizar e/ou apresentar às partes interessadas a eficácia das medidas adotadas na redução dos impactos ambientais (presentes e futuros) e na atuação social em atendimento aos seus objetivos de responsabilidade social. Os indicadores de desempenho são úteis para suprir tal necessidade, uma vez que eles sintetizam as informações quantitativas e qualitativas que permitem a visualização da eficiência e da efetividade da empresa no uso dos recursos disponíveis para a geração de um produto final, do ponto de vista econômico, ambiental e social. (Cantarino, 2003). Eles também auxiliam na definição das metas e dos caminhos a serem trilhados para o alcance e a manutenção da sustentabilidade empresarial.

Cientes da importância deste instrumento para a gestão empresarial, os autores têm desenvolvido pesquisa e apoiado empresas na definição, implementação e relato de indicadores de desempenho que possibilitem gestores de empresa avaliá-lo ao longo do tempo e comparativamente ao de outras empresas. Neste artigo são apresentados critérios que os autores consideram necessários para a definição e implementação dos indicadores de desempenho nas empresas.

\section{IDENTIFICAÇÃO E COMUNICAÇÃO DO DESEMPENHO}

Segundo Brown (2000), as partes interessadas estão solicitando informações adicionais ao desempenho financeiro da empresa. Os principais questionamentos às atividades empresariais são (Keeble at al, 2002):

- Investidores estão procurando evidências de boa governança empresarial, considerando particularmente a estratégia do negócio e a efetividade de sua gestão de risco; - Clientes estão arguindo sobre a origem do produto, isto é, quem o fez e o que ele contém;

- Empregados estão interessados em trabalhar em empresas que visivelmente considerem sua responsabilidade para com o empregado, a sociedade e o ambiente;

- Governos e sociedade civil estão aumentando a pressão para que empresas divulguem seu desempenho social e ambiental.

O Capítulo 30 da Agenda 21 estimula o comércio e a indústria a comunicar seu desempenho ambiental e a reportar "anualmente sobre seus registros ambientais, tanto quanto seu uso de energia e recursos naturais" e "sobre a implementação de códigos de conduta promovendo melhores práticas ambientais".

A nova visão de sustentabilidade empresarial é a da integração do desempenho econômico, social, ambiental, de segurança e de saúde do trabalhador, que são indissociáveis, na nova visão de negócios. Há crescente demanda para que os relatórios de desempenho econômico das empresas publicadas anualmente sejam complementados com informações que demonstrem as responsabilidades e compromissos da empresa para manutenção e ou melhoria social e ambiental assim como a sua visão de futuro a longo prazo (que necessariamente perpassa pela visão de sustentabilidade).

Empresas, dentre as quais destacam-se as transnacionais e as multinacionais, estão implementando esforço para reportar anualmente seu desempenho em relação às questões 
econômicas, sociais, de segurança e de meio ambiente através da publicação periódica do relatório de responsabilidade social / sustentabilidade. (Holliday, 2003) Eles são instrumentos de benchmark e de informação do seu desempenho anual às partes interessadas. Os relatórios voltados apenas para a melhoria da imagem (informações expositivas) estão sendo refinados e passam a conter informações que refletem a efetiva responsabilidade social e ambiental da empresa. A melhoria de imagem é uma conseqüência do reporte e não um fim em si mesmo.

Consciente destes requisitos surge então algumas questões sobre o desenvolvimento, acompanhamento, comparação análise e reporte dos indicadores que servirão de instrumentos para a demonstração do compromisso com a responsabilidade social e ambiental.

- Como definir os indicadores e estabelecer métodos de acompanhamentos de forma consistente?

- Como elaborar um relatório que propicie bases para comparação, troca de experiências (Benchmarking) com outras empresas e possam ser auditados?

- Como conciliar o relatório elaborado de forma voluntária seguindo as orientações de organizações internacionais, como o GRI, e aqueles requeridos por diplomas legais municipais, estaduais e federal, por vezes contraditórios, sem haver multiplicidade de esforços ou burocratização?

\section{Selecionando e Definindo Indicadores}

Há tendência de selecionar, dentre os indicadores propostos por organizações líderes na proposição de indicadores para Relatórios de Sustentabilidade e por organizações internacionais de normalização, aqueles que tenham características semelhantes aos usados por empresas do mesmo setor (Keeble et al. 2002). Ressalta-se que os indicadores necessitam refletir as realidades do "negócio", os valores e a cultura da organização (Arthur D. Little, 2003), ser compreensíveis pelas partes interessadas e úteis para ambos. Faz-se necessário ter uma metodologia que permita selecionar indicadores de sustentabilidade empresarial corporativos.

A metodologia proposta para a eficaz seleção e definição de indicadores segue os tópicos considerados fundamentais para uma eficiente gestão corporativa: compreensão dos valores e aspirações empresariais; identificação das partes interessadas e das questões que estas consideram relevantes que merecem ser consideradas; seleção dos indicadores a serem apropriados e das metas a serem alcançadas, coleta de dados, apropriação dos indicadores selecionados, análise da aplicabilidade dos indicadores selecionados; apresentação dos resultados às partes interessadas e avaliação da adequação dos indicadores.

Os indicadores precisam ser avaliados quanto aos critérios considerados essenciais à sua escolha, utilização e consistência (Cantarino, 2003). Estes critérios (Figura 1) foram sugeridos através do cruzamento e análise dos critérios estabelecidos por organizações líderes na concepção de critérios de sustentabilidade, como: Banco Mundial, Global Reporting Initiative (GRI), Environmental Protection Agency (EPA), CSIRO, Ministry for the Environment (MFE), FEM\&EEA, International Standart Organization (ISO), DOW JONES, etc. 
Figura 1: Critérios para a seleção e avaliação da adequação dos indicadores

\begin{tabular}{|l|c|c|}
\hline \multicolumn{1}{|c|}{ Escolha } & Utilização & Consistência \\
\hline$\checkmark$ Viabilidade & $\checkmark$ Sensibilidade & $\checkmark$ Relevância \\
$\checkmark$ Simplicidade & $\checkmark$ Espacialidade & $\checkmark$ Discriminatório \\
$\checkmark$ Cientificamente aceitável & $\checkmark$ Temporalidade & $\checkmark$ Balanceado \\
$\checkmark$ Clareza & $\checkmark$ Confiabilidade & $\checkmark$ Verificabilidade \\
$\checkmark$ Diretamente ligado ao objeto & $\checkmark$ Facilidade & $\checkmark$ Comparabilidade \\
\hline
\end{tabular}

Fonte: Cantarino, 2003

Nesta metodologia para a seleção de indicadores há uma quantificação com a atribuição de valores que variam de zero até um (1) para cada fator dos três (3) critérios referenciados na Figura 1, . 0 indicador é considerado efetivo para utilização se obtiver pelo menos nota 3 em cada critério, em uma escala que vai de zero (indicador reprovado) a 5 (melhor indicador). Todos os números obtidos são acompanhados por uma pequena descrição dos principais aspectos a ele relacionados.

De posse dos indicadores considerados adequados para utilização, a última etapa para a seleção e definição dos indicadores estratégicos é a verificação do seu grau de utilidade, que é definido junto aos tomadores de decisão conforme apresentado pela Figura 2. 0 indicador será considerado estratégico se for útil para muitos tomadores de decisão. 0 conjunto final de indicadores deve resultar num balanceado conjunto de indicadores que reflitam os interesses das diferentes partes interessadas (Keeble, et al. 2002).

Figura 2: Definição dos Indicadores Estratégicos

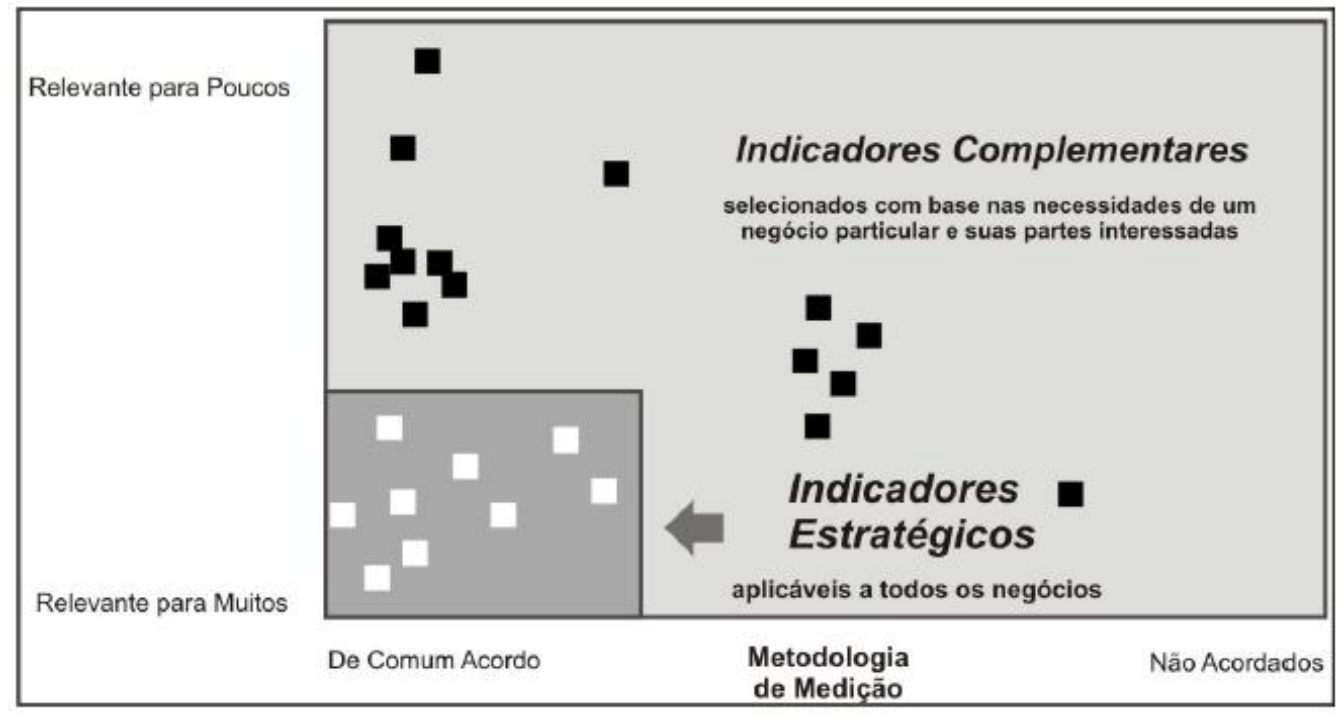

Fonte: Arthur D’Little

Com os indicadores selecionados e definidos, começa a fase de implementação que dependendo do porte e complexidade da empresa, pode ser dividida em etapas ou através da aplicação de um projeto piloto.

\section{Implementando Indicadores}


A fase de implementação é decisiva para assegurar que a cultura de sustentabilidade seja inserida e consolidada dentro da empresa. Segundo Keeble et al. (2002), o fator crítico para a implementação dos indicadores é assegurar a transferência da responsabilidade para a gerênciada empresa. 0 comando da empresa necessita entender como os indicadores podem ser usadoscomo instrumento para auxiliar os tomadores de decisão e assim, melhorarem a sustentabilidade empresarial.

A implementação dos indicadores pressupõe o envolvimento de toda a força de trabalho e principalmente da alta direção no que se refere à integração do negócio direcionando-o à sustentabilidade. Estes necessitam estar conscientes de que além do desempenho financeiro empresarial, a qualidade do desempenho social, ambiental, de saúde e segurança do trabalhador e do produto, também são objeto de gestão empresarial e conseqüentemente de mensuração através de indicadores de desempenho.

Deve se incorporar estes conceitos em cada integrante da força de trabalho (neste ponto ressalta-se que estarão envolvido também os contratados terceirizados e fornecedores). A responsabilidade pelo sucesso é de todos. Ao final, os dados que compõe os indicadores serão medidos, discutidos e registrados.

A avaliação integrada dos indicadores assim como a evolução dos índices que os representam, fornecem instrumentos para que se avalie a causa do avanço ou do retrocesso do seu desempenho. Estes devem ser claramente identificados, assim como, as iniciativas ou programas relacionados aos indicadores, de modo a corrigir eventuais desvios em tempo hábil para a manutenção da sustentabilidade empresarial ao longo do tempo. Com este sistema implementado, as decisões serão tomadas com base no conjunto de indicadores e nas suas inter-relações.

Há uma indissociabilidade entre os diversos indicadores. Isto mostra que embora o objetivo estratégico permaneça sendo a sustentabilidade empresarial, o seu alcance no atual cenário mundial de mudança de paradigma perpassa pela incorporação das questões sociais, ambientais, de saúde e segurança do trabalhador. Em outras palavras, visualiza-se uma nova forma de se fazer negócio que permite ganhos mais significativos para todas as partes interessadas. Os indicadores que mostram o desempenho empresarial relativo a estas questões são instrumentos úteis para prover os gestores empresariais de alto grau de informação do progresso de seu negócio e da adequação deste a sua política corporativa. Mas é essencial o cuidado para que os indicadores reflitam efetivamente o que se espera deles sustentabilidade empresarial.

\section{Relatando o Desempenho}

Os indicadores de desempenho são instrumentos úteis para a gestão da empresa e também para informar às partes interessadas sobre o desempenho da empresa. Apresentou-se na introdução deste artigo alguns exemplos de que as partes interessadas, dentre as quais os agentes do mercado de capitais passam a considerar a responsabilidade social e o desempenho ambiental empresarial para a tomada de decisão. Atualmente, a forma usual de divulgação do desempenho é através de publicação no relatório sócio-ambiental anual da empresa.

Embora boa parte das grandes empresas já reporte de alguma forma o seu balanço sócioambiental, a forma de apresentação e a sua abrangência ainda suscita alguns questionamentos. Como exemplo, tomamos a análise realizada pela empresa de consultoria 
Arthur D'Little em nove empresas de porte mundial que atuam no setor de energia (Figura 3), considerando seus relatórios sócio-ambientais publicados no ano de 2001. Devido à questão de confidencialidade, os nomes das empresas foram preservados e não serão apresentados. A descrição dos requisitos e as considerações adotadas são apresentadas no anexo deste artigo.

Figura 3: Análise dos Indicadores reportados publicamente por empresas de energia de porte mundial

\begin{tabular}{|c|c|c|c|c|c|c|c|c|c|c|}
\hline Extrato da Análise & $\mathbf{A}$ & B & $\mathrm{C}$ & D & $\mathbf{E}$ & $\mathbf{F}$ & G & HH & II & $\boldsymbol{\Sigma}$ \\
\hline Definição dos indicadores & 0 & 0 & D & D & D & D & 0 & - & $\mathrm{O}$ & 4,5 \\
\hline Indicadores por área de negócios & ? & D & 9 & D & ? & 0 & O & - & 9 & 6,0 \\
\hline Processos/Atividades incluídas & - & 0 & 0 & 0 & $\mathrm{O}$ & $\mathrm{O}$ & - & - & 0 & 3,0 \\
\hline Discussão dos indicadores & 0 & 0 & 0 & 0 & 0 & 0 & 0 & O & D & 7,0 \\
\hline Exemplos de Casos & D & 0 & D & 0 & 0 & 0 & 0 & O & 0 & 5,5 \\
\hline Série Histórica & - & 0 & - & $\mathrm{O}$ & - & - & - & - & - & 7,0 \\
\hline Clareza dos Resultados & - & - & ○ & 0 & ○ & ○ & - & - & ○ & 7,0 \\
\hline Abrangência dos Indicadores & - & D & ○ & D & - & D & • & $\bullet$ & D & 7,0 \\
\hline Apresentação de Metas & D & 0 & D & 0 & 0 & 0 & 0 & 0 & D & 3,5 \\
\hline Apresentação de metodologia & 0 & $\mathrm{O}$ & 0 & $\mathrm{O}$ & $\mathrm{O}$ & 0 & 0 & 0 & 0 & 2,0 \\
\hline$\Sigma$ & 9,0 & 2,0 & 6,0 & 2,5 & 7,5 & 5,0 & 9,0 & 6,5 & 5,5 & \\
\hline
\end{tabular}

Como podemos notar há algumas diferenças significativas entre os relatórios analisados. Pela atribuição de valores, notamos que as metas, as condições de contorno (inclusão / exclusão) e diretrizes referentes aos indicadores não são ainda usualmente apresentadas.

As diferenças ocorrem devido ao fato de que poucas empresas estão seguindo as orientações de uniformização de relatórios conforme proposto, por exemplo, pelo GRI. De outra forma, notamos que o grau de detalhamento das informações referentes às questões de meio ambiente e sustentabilidade possuem bastante diferenciação. Enquanto que algumas empresas apresentam as questões com comprometimento da sua discussão, outros ainda estão apresentando seus relatórios como "green glossies" (Skilliu \& Wennberg, 1998).0s caminhos através de responsabilidade social e ambiental demonstram a necessidade de que as empresas dêem mais um passo. As expectativas sobre o valor das empresas estão se ampliando e indicadores relacionados aos temas de ética, trabalho infantil, diversidade, ciclo de vida, biodiversidade, tecnologia de inovação, direitos humanos e ecoficiência começam a despontar.

Partindo de premissa que só se gerencia aquilo que pode ser medido, os resultados fornecidos pelos indicadores relacionados aos temas mencionados se constituem em uma oportunidade única para a agregação de valor ao negócio, através de demonstração de uma visão ampla e responsável de gestão. 
Um exemplo clássico é das empresas que fazem parte do Índice Dow Jones de sustentabilidade (DJSI), estas obtiveram uma valorização média para suas ações de $22 \%$ acima do valor de seus concorrentes no segmento que não participam do DJSI, durante todo o período que vai de 1993 a dezembro de 2002.

No Brasil com a criação do primeiro fundo de investimento socialmente responsável, o Ethical Fund do ABN-ANRO, a expectativa é que o mesmo evento de valorização aconteça. A lógica é muito simples, empresas transparentes, responsáveis e com visão de longo prazo transmitem mais confiança para todas as partes interessadas. Mas, ressalta-se a importância de que os indicadores utilizados para relatar seu efetivo desempenho a terceiros sejam criteriosamente selecionados através de metodologia robusta para a seleção e apropriação destes. Pois a parte interessada busca conhecer os resultados efetivos de desempenho sustentável e não apenas a intenção e/ou propaganda de realização de projetos de sustentabilidade.

\section{CONCLUSÃo}

O momento atual é de mudança de paradigma, pois os empresários ainda continuam perseguindo o lucro, mas a forma de se realizar os negócios e obter a desejada recompensa está passando por profundas transformações, onde aspectos tais como a responsabilidade social e ambiental, dentre outras, também passam a ser relevantes.

O caminho para a sustentabilidade empresarial necessita de instrumentos que efetivamente demonstrem a sua evolução. O desenvolvimento de indicadores que demonstrem a viabilidade, a confiabilidade e a efetividade operacional das empresas é fundamental para apoiá-las na caminhada para posições de liderança setorial. Salienta-se a necessidade de que seus relatórios anuais sejam claros, objetivos e abrangentes, voltados para o balanço de suas atividades.

Apresentamos aqui os critérios recomendáveis para a definição consistente de indicadores que apoiem na gestão e na comunicação do desempenho empresarial às partes interessadas. Ressalta-se a importância de selecionar entre os indicadores a serem apropriados pelas empresas, os que serão utilizados apenas para a sua gestão operacional e os que serão utilizados para demonstrar a todas as partes interessadas o efetivo desempenho da empresa. Propomos que os índices que compõem os indicadores sejam analisados comparativamente considerando os setores, o tamanho da atividade empresarial e os locais em que elas se desenvolvem, durante o processo de benchmarking entre as empresas.

Análise realizada pelos autores identificou que nos relatórios atualmente publicados, as informações referentes à questão responsabilidade social se encontram, na sua maioria, em forma embrionária, enquanto que as questões de meio ambiente, saúde e segurança são reportadas principalmente por indicadores reativos (aqueles que retratam um desempenho já alcançado).

Na nova visão de sustentabilidade empresarial o reporte dos indicadores reativos não é eliminado, pois os resultados alcançados suportam os novos passos a serem tomados. Entretanto, aqueles indicadores devem vir acompanhados de indicadores pró-ativos (aqueles que medem ações tomadas de modo antecipado, tais como treinamento ou investimento, com os quais se espera alcançar um determinado resultado), para que as partes interessadas também possam visualizar o potencial de desempenho futuro das empresas.

\section{REFERÊNCIAS BIBLIOGRÁFICAS}


AMARAL, S.P. Estabelecimento de Indicadores e Modelo de Relatório de Sustentabilidade Ambiental, Social e Econômica: uma Proposta para a Indústria de petróleo Brasileira. Tese de Doutorado. Rio de Janeiro: COPPE/UFRJ, 2003.

BARATA, M.M. L. Aplicação de uma Estrutura Contábil para Apropriação de Custos Ambientais e Avaliação da sua Influência no Desempenho Econômico das Empresas. Tese de Doutorado. 225 p. Rio de Janeiro: COPPE/UFRJ, 2001.

. Chamas,C.I. Gestão Ambiental e Desempenho Empresarial. Curitiba, ISKM 2003.

BROWN, D. The Accountable Business: Managing Corporate Responsability in Pratice. Arthur D. Little. 2000.

Cantarino, A. A. A. Indicadores de desempenho ambiental como instrumento de gestão e controle nos processos de licenciamento ambiental de empreendimentos de exploração e produção de petróleo nas áreas offshore. Tese de Doutorado. 404 p.

CGLRM - Council of Great Lakes Research Managers, 1991. A proposed Framework for Developing Indicators of Ecosystem Health for the Great lakes Region. International Joint Commission, Windsor, Ontario. 47 pp.

CSIRO - A Guidebook to Environmental Indicators. 1998, Australia. 20 pp.

EPA - United States Environmental Protection Agency - Evaluation Guidelines For Ecological Indicators. Editado por Jackson, L. E.; Kurtz, J. C.; Fisher, W. S. April 2000, pag 1-1 a 1-4. Washington DC

EYLES, J. and Cole, D. 1995. Human Health in Ecosystem Health: Issues and Meaning and Measurement. Monograph prepared for the Great Lakes Science Advisory Board, International Joint Commision, Windsor, Ontario. 145 pp.

FEM \& FEA - Federal Environment Ministry, Bonn and Federal Environmental Agency, Berlim. A Guide to Corporate Environmental Indicators. December, 1997. Pg 9.

FERREIRA, A. C. S. Uma Contribuição para a Gestão Econômica do Meio Ambiente - Um Enfoque de Sistema de Informações. Tese de Doutorado, São Paulo: FEA/USP, 1998.

GRI - Global Reporting Initiative - Sustainability Reporting Guidelines on Economic, Environmental, and Social Performance. June 2000. Boston - USA. Pg 16-18

WORLD BANK - Pollution Prevention and Abatement Handbook - Part II. Indicators of Pollution Management. Pg 3 - 4. 1997.

GRILLO RAMOS, M.L. Balanço Ambiental: Instrumento de Contabilização e Divulgação das Relações das Empresas com o Meio Ambiente. Dissertação de Mestrado. Rio de Janeiro: COPPE/UFRJ, 1999.

GUARNIERI, L.S. A Contabilidade e o Desenvolvimento Sustentável - Um Enfoque nas Informações Contábeis, Sociais e Ambientais da Indústria Siderúrgica. Dissertação de mestrado em Ciências Contábeis, Universidade do Estado do Rio de Janeiro, 2001.

HOLliDAY, C.; Schimidheiny, S.; Watts, P. Cumprindo o prometido: casos de sucesso de desenvolvimento sustentável. Rio de Janeiro: Campus, 2002. 
ITFM - Intergovernmental Task Force on the Monitoring Water Quality. Water Quality Monitoring in the United States - Technical Appendixes. 1993 Report. Prepared by ITFM, Advisory Committee on Water Information Coordination Program, Washington, D.C. January 1994.

KEEBLE, J. J.; Topiol, S. and Berkley, S. Using indicators to measure sustainability performance at a corporate and project level.

LA ROVERE, E. L.; Barata, M.M.L.. Indicadores de Ecoefiicência para a Petrobras. Rio de Janeiro, COPPE, 2003.

MFE - Ministry for the Environment - Proposed Indicators for the Environmental Effects of Energy. June 2000, pag 6. Wellington, New Zealand.

RIBEIRO, M.S. Contabilidade e Meio Ambiente. Dissertação de Mestrado. São Paulo: FIPECAFI/USP, 1992.

SKILliU, A and WENNBERG, U. Continuity, Credibility and Comparability: Key challenges for corporate environmental performance measurement and communication. The International Institute for Industrial Environmental Economics at Lund University. A report commissioned by the European Environment Agency. February 1998. 85 p.

\section{ANEXO}

1. Definição dos Indicadores - Informa o significado do indicador e sua finalidade

Valor 1- Mais de $50 \%$ dos indicadores reportados apresentam uma definição clara do seu significado e finalidade Valor 0,5 - Menos de $50 \%$ dos indicadores reportados apresentam uma definição clara do seu significado e finalidade

Valor 0 - Os indicadores reportados não são definidos 2. Indicadores por áreas de negócio Apresenta resultados por áreas de negócio da empresa

Valor 1 - Mais de 50\% dos indicadores reportados são apresentados corporativamente e pelas áreas de negócio da empresa

Valor 0,5 - Menos de 50\% dos indicadores reportados são apresentados corporativamente e pelas áreas de negócio da empresa

Valor 0 - Os indicadores reportados não são apresentados por área de negócio, mas apenas corporativamente

3. Processos/ atividades incluídas - Apresenta as fontes de emissão, os processos ou atividades que foram incluídos no acompanhamento dos indicadores

Valor 1 - Para mais de 50\% dos indicadores reportados são apresentados os processos e/ou atividades que estão incluídas na sua medição, como por exemplo: flare, craqueamento catalítico, águas de resfriamento.

Valor 0,5 - Para menos de 50\% dos indicadores reportados são apresentados os processos e/ou atividades que estão incluídas na sua medição

Valor 0 - Para os indicadores reportados não são apresentados os processos e/ou atividades 
que estão incluídas na sua medição.

\section{Discussão dos Indicadores - Realiza a análise dos resultados apresentados pelos} indicadores

Valor 1- Mais de 50\% dos resultados dos indicadores reportados são discutidos e as justificativas apresentadas.

Valor 0,5 - Menos de 50\% dos resultados dos indicadores reportados são discutidos e as justificativas apresentadas.

Valor 0 - Os resultados dos indicadores reportados não são discutidos

5. Exemplos de casos - Fornece exemplos de casos ou ações tomadas para obter um melhor desempenho em relação a aquele indicador

Valor 1 - Mais de 50\% dos resultados dos indicadores reportados são exemplificados através de estudos de caso.

Valor 0,5 - Menos de 50\% dos resultados dos indicadores reportados são exemplificados através de estudos de caso

Valor 0 - Os resultados dos indicadores reportados não são exemplificados

6. Série Histórica - Acompanha o mesmo indicador, com o mesmo método por pelo menos 3 anos consecutivos

Valor 1 - Mais de 50\% dos indicadores reportados são acompanhados por pelo menos 3 anos consecutivos com a mesma metodologia de medição.

Valor 0,5 - Menos de 50\% dos indicadores reportados são acompanhados por pelo menos 3 anos consecutivos com a mesma metodologia de medição.

Valor 0 - Os indicadores reportados são acompanhados por menos 3 anos consecutivos ou houve mudança na metodologia de medição.

7. Clareza dos Resultados - Os resultados são apresentados em forma numérica e com as respectivas unidades

Valor 1 - Mais de 50\% dos resultados dos indicadores reportados são apresentados com valores claros em forma de tabelas e/ou gráficos

Valor 0,5 - Menos de 50\% dos resultados dos indicadores reportados são apresentados com valores claros em forma de tabelas e/ou gráficos

Valor 0 - Os resultados dos indicadores reportados não são apresentados com valores claros, mas apenas as ordens de grandeza.

8. Abrangência dos Indicadores - Cobre os temas referentes ao meio ambiente (efluentes, emissões, resíduos, vazamentos, recursos ambientais), saúde, segurança e responsabilidade social

Valor 1 - 0 relatório anual apresenta indicadores que abrangem mais de 50\% dos seguintes temas: Meio Ambiente (emissões, efluentes, resíduos, vazamentos, consumo de recursos 
ambientais), Saúde, Segurança; Responsabilidade Social.

Valor 0,5 - 0 relatório anual apresenta indicadores que abrangem menos de 50\% dos seguintes temas: Meio Ambiente (emissões, efluentes, resíduos, vazamentos, consumo de recursos ambientais), Saúde, Segurança; Responsabilidade Social.

Valor 0 - 0 relatório anual apresenta indicadores que abrangem apenas um dos temas acima mencionados.

\section{Apresentação de Metas - Apresenta metas referentes aos indicadores para os anos subsequentes}

Valor 1 - Para mais de 50\% dos indicadores reportados são apresentadas metas para os anos subseqüentes.

Valor 0,5 - Para menos de 50\% dos indicadores reportados são apresentadas metas para os anos subseqüentes.

Valor 0 - Para os indicadores reportados não são apresentadas metas.

\section{Apresentação da Metodologia - Apresenta as diretrizes adotadas para acompanhar os indicadores}

Valor 1 - Para mais de 50\% dos indicadores reportados são apresentadas as metodologias adotadas.

Valor 0,5 - Para menos de 50\% dos indicadores reportados são apresentadas as metodologias adotadas.

Valor 0 - Não são apresentadas as metodologias adotadas para acompanhar os indicadores reportados. 